\title{
Algorithm Optimization on Slab Re-storage of In-stock and Out-stock of Hot Rolling Plant

\author{
Li You
}

\author{
Metallurgical Automation Research and Design Institute, BeiJing, 100071, China
}

\begin{abstract}
Key Words: slab storage; algorithm of slab re-storage; mixed optimization; optimization algorithm of DPSO
\end{abstract}

\begin{abstract}
In order to solve the plan formulation of slab storage, this paper proposes the problem of dividing out-stock of slab storage into optimization measure of slab storage and decision combined optimization of slab re-storage. This paper has respectively established optimized decision model of slab in-stock and out-stock as well as optimal decision model for slab re-storage, constructs one kind of mixed genetic algorithm to make quick solution, it uses actual production data to make research on model and algorithm feasibility, demonstrates the effectiveness of model and algorithm of this paper, it adopts optimized algorithm of DPSO suitable for model to make solution. The test result indicates that model and algorithm of this paper are effective and practicable, crane stroke and amount of slab re-storage have been greatly reduced, which can enhance automatic level of slab storage.
\end{abstract}

\section{Introduction}

In the process of iron production, hot rolling procedure organizes production according to plan, the essence of plan content can meet production procedure[1,2,3] of slab restricted by continual rolling process in aspects such as width, thickness, iron variety and weight etc. At present, for the optimized management of slab storage, especially for research on out-stock and slab re-storage is very little, whether there is overlap [4] between different rolling positions, it can divide research on slab re-storage of current literature into 2 kinds: corresponding relations on rolling position and slab in stock of one-to-many and many-to-many. It uses optimal slab re-storage as the sub-target of plan formulation of slab out-stock, using hierarchical approach to establish 2 optimal decision models of slab re-storage and out-stock as well as designs one kind of optimization algorithm of DPSO with high efficiency[5], it designs heuristic algorithm [6] based on section dynamic plan. As for the slab re-storage problem, it designs one kind of improved partheno-genetic algorithm[7], for the container transposition problem, it designs one kind of heuristic algorithm of neighborhood search [8], dividing container transposition into matching of stowage location, movement plan, task sequence to make solution. The above research outcomes are mainly focus on slab, coil, container transposition; it has certain instructive effect on the research of this paper.

This paper uses conditions of many-to-many as research content, on the basis of the above research, it establishes the optimal slab re-storage model of many-to-many corresponding relations of rolling position and slab in stock, considering characteristic of continual distribution for slab of the same specification, it establishes constraint satisfaction model for optimal decision of steel pipe in-stock, on the basis of solution algorithm, for the characteristic of in-stock steel pipe with many specifications, it creates one kind of self-adaptation chaos genetic algorithm, using natural coding way, dynamically adjusts intersection and variation probability of algorithm online and adopts chaos genetic algorithm as variation operator. It establishes plan formulation model and algorithm of slab out-stock, changes value way and speed change of particle, it can quickly make the solution.

\section{Problem Description and In-stock, Out-stock Modelling}

\subsection{Problem Description}

Slab re-storage means there is one piece of several pieces of slabs beyond of rolling plan on the 
surface of slab in the rolling plan, when this slab is out of stock, it needs to transfer the slab onto it to the other slab position, here it uses hoisting and retraction as slab re-storage foe once. Usually, every rolling position has several exchangeable slabs matching with it in the slab storage, meanwhile, in order to guarantee even stress of roller, slab distribution in the rolling plan has certain consistency, it has the continual distribution condition of slab with the same specification and nature, it is regarded as slab specification group, which constructs many-to-many relations between slab needed by hot rolling plan and slab in the slab storage.

The formulation of slab out-stock is focuses on demand of each piece of slab in hot rolling production line, it chooses suitable slab provided to hot rolling production line from stock of slab storage, so that it can guarantee smooth progress of steel-making, continuous casting and hot rolling. There is hundred of piece of slabs in the slab storage, there are many slabs with the same specifications, and operation of slab in-stock and out-stock is very frequent, it needs many times movement and slab re-storage of slab by crane; this makes formulation of out-stock plan as difficult problem of NP.

Meanwhile, the problem of out-stock formulation needs to consider amount of slab re-storage and crane stroke as well as many restrained conditions, it is one complicated optimized and combination problem. Because it is target optimization problem, it has large scale and many constrained conditions, the solution is relatively difficult. On condition of guaranteeing solution of optimized problem and suitable for application of site production, it adopts hierarchical approach, it uses out-stock plan to solve this complicated multi-target optimization problem, dividing into 2 optimal combination problems: slab optimization decision of out-stock and optimal slab re-storage of slab, respectively establishes optimal decision model. In the system design, management and slab stacking of slab re-storage adopt logic sub-region and stacking of rolling plan, that means the same slab is stacked in the same region when slab storage makes stacking, the slab belongs to the same formulation plan should be possibly stacked together, optimal management system of slab storage adopts 3 coordinate of 3 dimensions to make positioning for slab, every piece of slab has its own storage number of 2 dimensions and slab storage number of 1 dimension to confirm position, position of out-stock roller table in the slab storage and crane position all adopt position number of 2 dimensions to mark it.

\subsection{Model establishment of slab in-stock}

As for each slab batch needs in-stock, in-stock decision chooses suitable storage position and slab position for every slab on condition of meeting in-stock position and choice principle of slab position, making slab in the whole in-stock batch have optimal out-stock effect for the chosen storage position and slab position, that is to choose optimal storage position and slab position for slab, this is one large-scale and complicated optimization problem, considering the main principle of slab in-stock, the slab in-stock principle established by this paper is as follows:

$$
\begin{aligned}
& P=\max \sum_{i=1}^{n u m} p_{i} \\
& P=\max _{j=1}^{m}\left(p_{i j}\right) \quad \mathrm{j}=1,2, \ldots, \mathrm{m} \\
& p_{i j}=q_{i j} k_{i j}\left(a_{i} S_{i j}+a_{2} w_{i j}+a_{3} T_{i j}+a_{4} R_{i j}+a_{5} D_{i j}+a_{6} N_{i j}+a_{7} H_{i j}+a_{8} F_{i j}\right)
\end{aligned}
$$

Of which, num is the number of slab in in-stock batch, Pi the condition accommodation of final slab position for slab $\mathrm{i}, \mathrm{Pij}$ accommodation value of confirming its optimal in-stock position $\mathrm{j}$ for every piece of slab $i, i=1,2, \ldots$, mun is the number of slab needs in-stock, $j=1,2, \ldots, m$ is the storage number of slab storage, a1 a8 are respectively the same rolling plan, slab width, thickness, hardness, out-stock time, empty storage position, mixed stacking, weight parameter of fixed matching plate. $q_{i j}, k_{i j}, S_{i j}, w_{i j}, T_{i j}, R_{i j}, D_{i j}, N_{i j}, H_{i j}, F_{i j}$ is respectively 0-1 function, 1 means the same thickness, width. 


\subsection{Model establishement of slab out-stock}

Optimized decision model of slab out-stock is to confirm the slab or slab assemble with the minimum slab re-storage number according to slab information, slab stock information, stacking information of each kind of slab in the lab plan in the hot rolling production line, the target is to minimize slab re-storage for the slab rout-stock plan with the same batch, this is one kind of combined optimization problem, this paper establishes the following optimization model for the decision problem of slab out-stock.

$$
\begin{gathered}
\left.P=\min \sum_{i=1}^{n} \sum_{j=1}^{m_{i}}\left\{D_{j}-\sum_{k=1, K U_{k}=K U_{j}}^{i=1} \min \left\{\max \left\{D_{j}-D_{k}\right\}, 0\right\}, 1\right\}\right\} \times \mathrm{x}_{\mathrm{ij}} \\
\text { St } \sum_{\mathrm{j}=1}^{\mathrm{m}} \mathrm{x}_{\mathrm{i}}=1 \\
\sum_{i=1}^{n} x_{i j}=1 \& 0
\end{gathered}
$$

Of which, $\quad x_{i j}=\left\{\begin{array}{cc}1 & \text { The I location selection plan provided blanks numbered } \mathrm{J} \text { slab } \\ 0 & \text { else }\end{array}\right.$ $\mathrm{i}=1,2, \ldots, \mathrm{n}, \mathrm{n}$ is the slab number in the slab plan $, \mathrm{j}=1,2, \ldots \ldots, \mathrm{mi}$, mi is the total number of target slab $\mathrm{i}$ in the belonging area of slab storage, $\mathrm{Dj}$ is the slab number of the $\mathrm{j}$ slab, Kuk is the storage number of the $\mathrm{k}$ slab.

\section{Mixed genetic algorithm of slab in-stock}

\subsection{Intersection and variation probability of self-adaptation adjustment}

According to the concentrated degree of adapt value to dynamically adjust the intersection probability Pc and variation probability Pm in group of each generation, it adopts the maximum adaptation value fmax in group of each generation, minimum adaptation degree value fmin, the average value fave to be used as the judgment standard on concentration degree of adaptation value. The probability adjustment formula of intersection and variation is as follows:

$$
\begin{gathered}
p_{c}= \begin{cases}p_{c 0}+\left(1-p_{c o}\right) \times f_{\text {min }} / f_{\text {max }} & f_{\text {min }} / f_{\text {max }}>\alpha, f_{\text {ave }} / f_{\text {max }}>\beta \\
p_{c o} & \text { else }\end{cases} \\
p_{m}= \begin{cases}p_{m 0}+\left(1-p_{m o}\right) \times f_{\text {min }} / f_{\text {max }} & f_{\text {min }} / f_{\text {max }}>\alpha, f_{\text {ave }} / f_{\text {max }}>\beta \\
p_{m o} & \text { else }\end{cases}
\end{gathered}
$$

\subsection{Chaos Optimization and Variation Operator}

Firstly, it chooses carrier type of chaos variable and uses lgoiscti reflection, of which, u is control parameter.

$x_{n+1}=u x_{n}\left(1-x_{n}\right)$

: Suppose the value range of individual gene in chromosome is [a, b], that is the coding range of chosen storage number, the method for chaos optimization variation is as follows:

(1) Find out the individual needs variation and confirm gene i needs variation operation in the individual and storage code ki corresponds to gene position, choose $\mathrm{x}=\mathrm{ki}$.

(2) Convert $x$ into number between $[0,1]$ according to the following formula, $x=(b-x) /(b-a)$

(3) Carry out chaos optimization reflection, adopt formula(9), it get $x^{\prime \prime}=u x^{\prime}\left(1-x^{\prime}\right)$

(4) $x^{\prime \prime}=a+x "(b-a)$; Convert $x^{\prime \prime} \quad$ into the number among gene value range, $x "=a+x "(b-a)$

(5) Make ki= $x^{\prime \prime}$, update the gene code of individual

(6) Judge variation operation is completed or not, then back to operation of genetic algorithm, otherwise, find out the next individual needs variation, back to (1) 


\subsection{Solution procedure of chaos genetic algorithm}

Solution algorithm of chaos genetic algorithm is indicated by diagram 1:

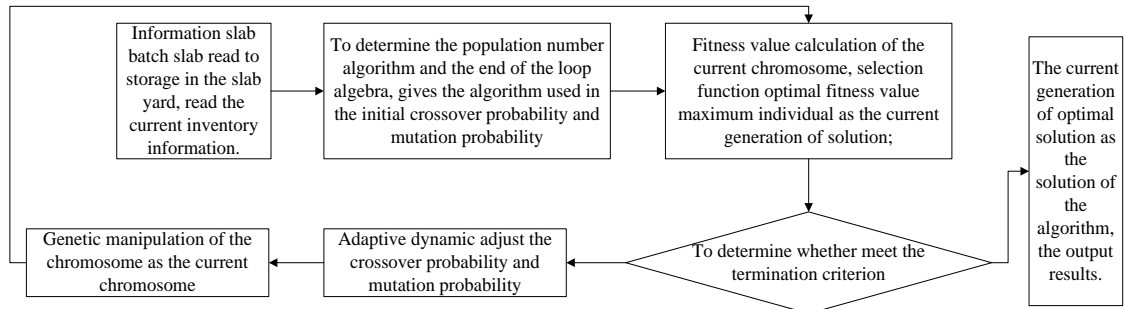

Diagram 1 Algorithm Procedure

\subsection{Calculation Example and Result}

In order to demonstrate feasibility of model and algorithm, it chooses some data from slab storage in the hot rolling plant, in-stock slab randomly chooses data of 18 pieces of slabs, stock data randomly chooses 15 storage with 80 piece of slabs as the storage condition can be chosen, suppose in-stock slab and chosen storage position belong to plan slab region, choice of model parameter is as follows: $\mathrm{a} 1=20, \mathrm{a} 2=2, \mathrm{a} 3=2, \mathrm{a} 4=2, \mathrm{a} 5=15, \mathrm{a} 6=10, \mathrm{a} 7=\mathrm{s}, \mathrm{a} 8=20$, the population number of chaos genetic algorithm is 20 , intersection probability $\mathrm{Poc}=0.95$, variation probability $\mathrm{Pmo}=0.10$, algebra of maximum cyclic iteration chooses 300 , result is indicated by diagram 2 , the optimal value of target function is 378/. The model and algorithm of this paper can completed for once and minimize the amount of slab storage in the whole batch slab. From diagram 2, we can see that model algorithm can quickly choose suitable storage position and slab storage for each piece of slab, with the increase of cyclic iteration, the optimization effect is very obvious.

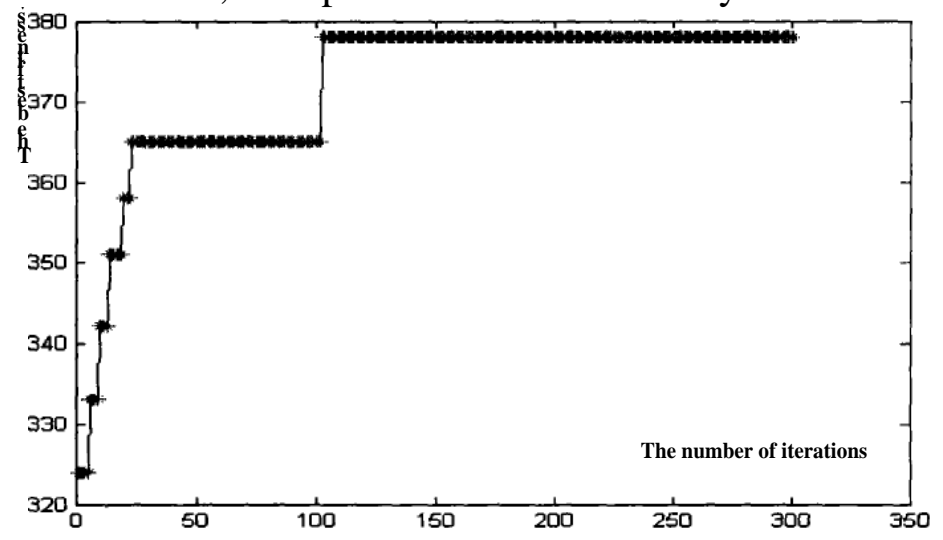

Diagram 2 Adaptation value of solution model for chaos genetic algorithm

\section{Solution Method on Plan Optimization Model of Slab Out-stock}

\subsection{DPSO}

For the above decision optimization model of the above mentioned slab out-stock, $\mathrm{n}$ is the number of slab in the slab extracting plan, Wi is the chosen slab assemble of the i slab, $\mathrm{Ni}$ is the slab number included by assemble $\mathrm{Wi}$, particle $\mathrm{Xi}=\{\mathrm{xi} 1, \mathrm{xi} 2, \mathrm{xi} 3, \ldots, \mathrm{xin}\}$ represents one solution of model, it chooses target function of decision optimization model of slab out-stock as the adaptation function of algorithm. This, Abestg is the optimal solution searched by algorithm. As for the optimal slab re-storage model of slab, algorithm parameter is changed as: Wi is the chosen slab assemble of the i slab with the same and minimum slab re-storage amount, target function (8) is used as function of adaptation value. Here it uses the above as basis to make operation on particle by the following formula:

$v_{i d}=v_{i d}+\operatorname{int}\left[c_{1} r\left(\right.\right.$ Pbest $\left._{i d}-x_{i d}\right)+c_{2} r_{2}\left(\right.$ Abest $\left.\left._{g d}-x_{i d}\right)\right]$ 
$x_{i d}=x_{i d}+v_{i d}$

$x_{i d}= \begin{cases}1 & \mathrm{x}_{\mathrm{id}}<1 \\ N_{i} & \mathrm{x}_{\mathrm{id}}>N_{l}\end{cases}$

Of which: $\mathrm{i}=\mathrm{I}, 2, \ldots \mathrm{m}, \mathrm{m}$ is the particle number of particle group, $\mathrm{d}=1,2, \ldots, \mathrm{n}, \mathrm{n}$ is the slab number in the slab extracting plan, $\mathrm{Ni}$ is the number of chosen slab in the i slab,; c1, c2 is learning factor and non-negative constant, it usually chooses 2, r1, , r2 is weight parameter, usually choosing random number between $[0,1]$.

Formula (10) and (11) are evolution mechanism of optimization algorithm for particle, formula (10) the value way of particle speed, formula (12) is the value way of particle, which is optimization choice mechanism in this algorithm. Formula (12) is the disposal for infeasible solution, it converts into feasible solution.

The step of DPSO on optimization algorithm to solve decision optimization model is indicated

by diagram 3.

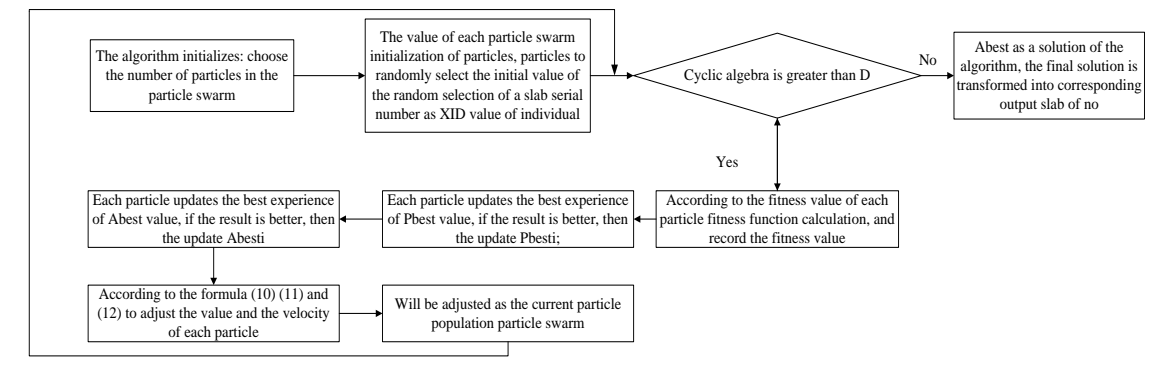

Diagram 3 Step of DPSO to solve decision optimization model

\subsection{Formulation process of out-stock plan}

The formulation process for slab out-stock is as follows:

Step 1: initialization, read the slab information of slab plan, stock information of slab storage and positioning information in slab storage.

Step 2: for the slab in the slab plan, confirm value range of each slab in the slab storage according to specification

Step 3: Use optimization algorithm of particle group to solve decision model of slab out-stock model and confirm each slab has the same and minimum chosen slab assemble of slab re-storage amount.

Step 4: Use the chosen slab with the same and minimum slab storage amount as input data, use optimization algorithm of particle group to solve optimal slab re-storage model of slab, and confirm the final out-stock slab of each slab in the slab extracting plan.

Step 5: Use expert system knowledge to judge the final result and used as plan result of out-stock, otherwise, rectify input data of stock slab, repeat (3)-(5).

\subsection{Calculation Example and Result}

In order to demonstrate effectiveness of model and algorithm, it randomly chooses 10 pieces of slab from the slab plan in the slab storage production data from hot rolling plant as out-stock slab, in-stock slab randomly chooses data of 18 pieces of slabs, stock data randomly chooses 15 storage with 80 piece of slabs as the storage condition can be chosen, it compares with optimization algorithm of scattered particle and basic genetic algorithm under MATLAB programming condition, respectively makes comparative study on optimization algorithm of scattered particle group and basic genetic algorithm in this paper, respectively uses optimization method of scattered particle and basic genetic algorithm to solve 2 models of this paper, the change result of adaptation value is indicated by diagram 4 and diagram 5 . The parameter choice of algorithm in this paper is as follows: the particle number of particle group is 10 , algebra of maximum cyclic iteration $g=1000$, intersection probability is 10 . The parameter choice of genetic algorithm is as follows: individual 
number of each generation chooses 10 , intersection probability is 0.95 , variation probability is 0.10 , cyclic iteration is 1000, the optimal value of adaptation function in genetic algorithm is repetitively 15 and 2489. Diagram 4 is respectively adaptation value curve of out-stock decision and optimization model to solve optimal slab re-storage for DPSO and GA., diagram 5 is respectively adaptation value curve of optimal slab re-storage model for DPSO and GA From the simulation study algorithm operation result, operation time of 2 kinds of algorithms is basically the same, about 2 seconds or so, but from diagram 4 and diagram 5, we can obliviously see that optimal speed and optimal solution designed by this paper are better than that of basic genetic algorithm.

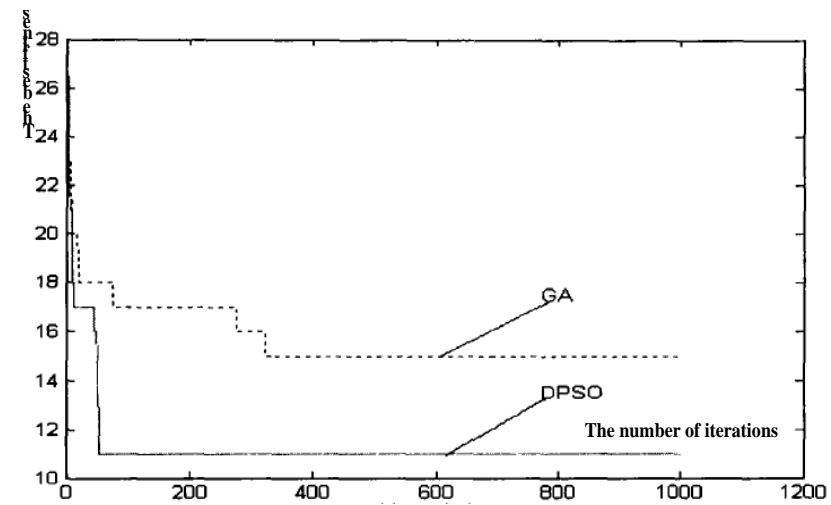

Diagram 4 Adaptation curve of slab out-stock decision model

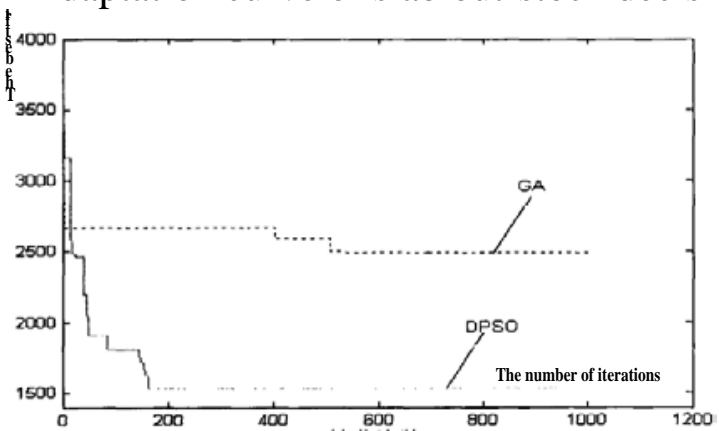

Diagram 5 Adaptation curve of optimal slab re-storage decision for slab

\section{Conclusions}

This paper establishes decision optimization model for slab in-stock, it also considers slab out-stock at the same time of slab in-stock. In order to effectively solve model, this paper uses characteristics of chaos optimization theory nature and genetic algorithm to construct chaos genetic algorithm, it adopts chaos optimization algorithm as variation operator of genetic algorithm to quickly search optimization solution of algorithm. This paper adopts actual production data to demonstrate model and algorithm, as well as compares result with human dispatch used on site, which shows the effectiveness of model and algorithm of this paper.

On the basis of analyzing characteristics of particle group optimization, it constructs scattered particle optimization algorithm suitable for solving these 2 models, it uses value way suitable for particle speed change of model and value way of particle to make quick solution. It uses site production data to make test on model and algorithm, according to the software system made by this algorithm, the application and test result indicate that model and algorithm of this paper is effective and practical, and crane stroke and amount of slab re-storage have been greatly reduced, which can enhance automatic level and production dispatch level of slab storage as well as save labor resources.

\section{References}

[1] Tang Lixin. Study on Iron Production Plan and Dispatch Based on Intelligence [J ] . Management Journal, 2005, 2(3): 263-267. 
[2] Li Yaohua etc.Out-stock Plan Formulation Model and Algorithm Study on Hot Rolling Slab [J].Computer Assemble and Production System

, 2006, 12(3): 377-381

[3] Li Tieke, Guo Dongfen. Batch Plan Model and Algorithm Based on Constrained Satisfied Hot Rolling [J].Control and Decision, 2007, 22(4): 389-393

[4] Li Yaohua,Hu Guofen,Wang Wei etc.Study on Systematic Production Plan Method of Iron Making, Continual Casting, Hot Rolling [J].Control Engineering, 2005,12(06):449-552

[5] Li Yaohua, Xu Lejiang, Hu Guofen etc. Decision Optimization Method of Slab In-stock Based on Chaos Genetic Algorithm [J].System Simulation Journal, 2005, 17(11):2620-2623.

[6] Li Dan, Wang Jing, Lv Zhimin. Decision Optimization Method Based on Slab In-stock of Hot Delivery and Hot Package ( J `.Information and Control, 2008,37(5):529-533.

[7] ) Zhao Qun, Wang Wei, Liu Quanli. Group Dispatch Model and Algorithm of Cold Rolling Sheet[J].Computer Assemble and Production System,2008,14(10):1957-1965. 。

[8] Wang Ling etc.Genetic Algorithm and Its Capacity Analysis [J].Control and Decision, 2005, 20(2):156-160.

[9] Dong Guangjing. Problem Model and Algorithm Study on Tube Blank In-stock [J].Industrial Engineering Management.201318 (6) : 32-39..

[10] Li Yaohua. Plan Formulation Model and Algorithm Study on Hot Rolling Slab. [J].Computer Assemble and Production System, 2006,12(3): 377-381. 